Review

\title{
Ionized or Total Magnesium levels, what should we measure?
}

\author{
Giuliana Scarpati ${ }^{1 *}$, Daniela Baldassarre ${ }^{1}$, Filomena Oliva ${ }^{1}$, Gabriele Pascale ${ }^{1}$, Ornella Piazza1 \\ 1 University of Salerno \\ * Correspondence: gscarpati@unisa.it
}

\begin{abstract}
Monitoring and measuring magnesium $(\mathrm{Mg})$ values are essential to prevent the development of numerous complications in perioperative medicine and critically ill patients. Although previous studies suggest that measuring free ionized magnesium (iMg) is more useful for estimating $\mathrm{Mg}$ status, clinicians currently rely on measurement of total serum magnesium to determine if supplemental magnesium is needed. In this review, we analyzed the recent literature to decide whether it is better to measure ionized serum $\mathrm{Mg}$ or total serum $\mathrm{Mg}$ when assessing magnesium status, whether iMg predicts clinical outcome, and what are the difficulties in measuring serum iMg levels in intensive care patients and perioperative medicine.
\end{abstract}

Keywords: Ionised Magnesium; Preoperative Medicine; ICU; Dysmagnesemia

\section{Ionized vs total magnesium levels in Intensive Care Patients}

Ionized magnesium (iMg) constitutes $50 \%$ of the total magnesemia and represents the electrophysiologically active portion. $10 \%$ of magnesium in the blood is complexed with anions, and the remaining $30-40 \%$ is bound to albumin [1].

Hypomagnesaemia occurs postoperatively in about $60 \%$, in $65 \%$ of patients admitted to medical intensive care (ICU) and in about $90 \%$ of surgical patients [2]. Although total Mg and ionized $\mathrm{Mg}$ are generally related in subjects in good health, the literature on $\mathrm{Mg}$ supplementation has been disparate [3-5]. iMg is rarely measured in a clinical setting [6], perhaps because it requires specialized equipment for measuring iMg on whole blood. Additionally, iMg values may be subject to individual factor interference. Very few studies have evaluated the relation between $\mathrm{tMg}$ and iMg in patients admitted to intensive care. Cardiovascular dysfunction and systemic inflammatory response syndrome in ICU patients are closely related to hypomagnesaemia [2].

Dysmagnesaemia is also common in critically ill patients and has been shown to be associated with a higher mortality rate and longer ventilatory support.[7-9]. Also, several studies report a correlation of dysmagnesemia with increased mortality, length of stay, and morbidity in ICU patients [1014]. Nonetheless, hypomagnesemia is frequently underdiagnosed. Hypomagnesemia is present in approximately $70 \%$ of adult patients admitted to the ICU.

Gastrointestinal disturbances and renal loss of $\mathrm{Mg}$ are the main causes of hypomagnesaemia in critically ill patients [15] (TABLE 1) they are a frequent cause of secondary hypokalemia and hypocalcemia leading to severe neuromuscular and cardiovascular clinical manifestations.(TABLE

2) 


\section{Gastrointestinal disturbances}

Nasogastric suction

Malabsorption syndrome

Bowel resection

Diarrhea

Malnutrition

Pancreatitis

Renal loss of Mg

Chronic parenteral fluid therapy

Osmotic diuresis

Hypercalcemia

Alcohol

Drugs

Metabolic acidosis

Renal diseases

Table 1 Causes of hypomagnesemia in ICU patients. 
Table 2 Clinical manifestations associated with hypomagnesemia and hypermagnesemia

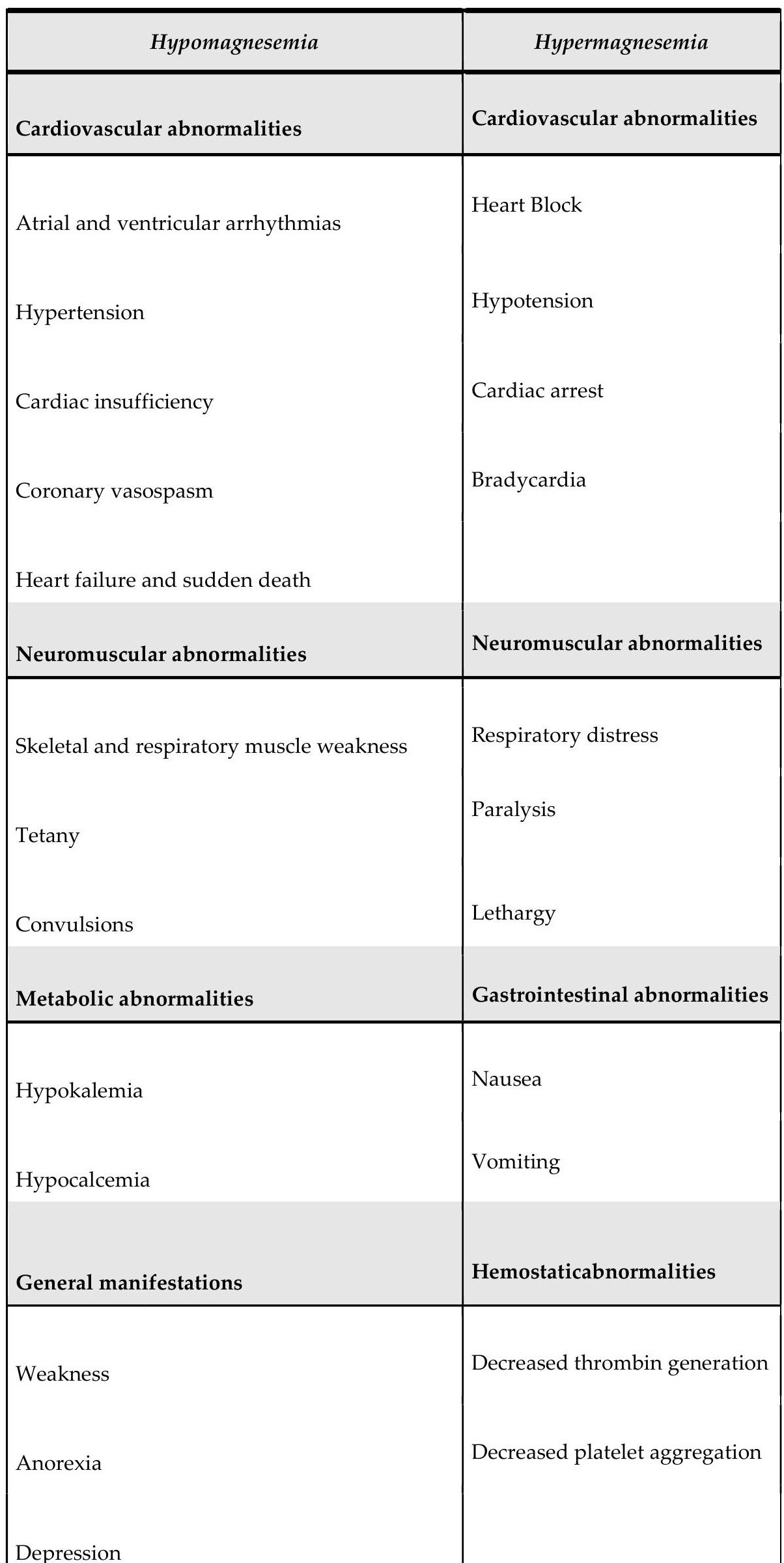


In particular, ICU patients receive different combinations of drugs and may have a reduced ability to eliminate the drug due to the reduced kidney and/or liver function that could influence Mg homeostasis [15].

Finally, diabetic ketoacidosis, starvation or alcoholism causing metabolic acidosis result in the renal loss of $\mathrm{Mg}$.

Del Gobbo et al. demonstrate that for every $0.49 \mathrm{mg} / \mathrm{dL}(0.2 \mathrm{mmol} / \mathrm{L})$ serum $\mathrm{Mg}$ reduction, there is a $30 \%$ increase in cardiovascular diseases [16]. Furthermore, other studies have reported a higher incidence of sepsis in ICU patients with hypomagnesaemia [10,11,17].

The relationship between hypomagnesaemia and mortality rate varies widely in the literature. Chernow et al. [18], Rubeiz et al. [19] and Safavi and Honarmand [20] found in their studies a higher mortality rate in patients with hypomagnesaemia than in normomagnesemic patients. While Guérin et al. [21] noted a higher ICU mortality rate among hypermagnesaemic patients with no difference in mortality between hypomagnesaemic and normomagnesemic patients. Finally, other studies [22,23] observed up to 3 times higher mortality rates in patients who develop ionized hypomagnesaemia while staying in the ICU. The average hospital mortality in all the studies was $24.3 \%$. Hypermagnesemia is present in $5 \%$ to $10 \%$ of critically ill patients [24]. Table 2 shows the clinical conditions associated with hypermagnesemia.

Several studies demonstrated that $\mathrm{iMg}$ is the only marker that specifically identifies patients with dysmagnesemia $[25,26]$. Besides, iMg monitoring of patients treated with Mg sulfate improves clinical outcomes. It can reduce the length of stay, and iMg monitoring reduces the risk of Mg toxicity in patients treated for $\mathrm{Mg}$ deficiency [26]. In critically ill patients, the correlation between iMg and total $\mathrm{Mg}$ has been demonstrated to be poor $[27,28]$. In fact, $69 \%-85 \%$ of critically ill patients with low total $\mathrm{Mg}$ results have been found to have normal iMg levels. Correcting Mg deficiency based on rapid, real-time measurement of iMg allows for a more accurate titration of Mg sulfate, but iMg must be directly measured; it cannot be calculated.

Yeh et Al. noted that using total Mg in clinical decision making can lead to over-treating patients with Mg sulfate and unnecessary repeat testing [27].

$\mathrm{iMg}$ is also vital for the intracellular regulation and transport of $\mathrm{Na}, \mathrm{K}$, and iCa [12]. So, rapid serial measurement of iMg is crucial for the management of electrolyte deficiencies [29]. Hypomagnesaemia and hypocalcemia are very common (up to $40 \%$ ) in patients with other electrolyte abnormalities [30], demonstrating that iMg, $\mathrm{Na}, \mathrm{K}$, and Ca should always be tested together for proper electrolyte near-normal. In fact, it was observed that the hypokalemia and hypocalcemia did not improve on intravenous $\mathrm{K}$ and $\mathrm{Ca}$ administration alone, it was only after $\mathrm{Mg}$ supplementation, the blood levels of $\mathrm{Na}, \mathrm{K}$ and Ca reached the near-normal levels [31].

\section{Ionized vs total magnesium levels in the perioperative setting}

The interest in magnesium and its biologically active form continues to be attentive despite conflicting data of the effect of magnesium supplementation on preventing perioperative adverse events or predicting health outcomes in surgical patients. A state of perioperative hypomagnesemia, not present before surgery, has been reported [32,33], considering the total serum magnesium easily measurable. The available data on the possible benefit of the magnesium supplementation in the perioperative period concern different aspects. 
The greatest attention has been pointed to the prevention of arrhythmias in high-risk patients undergoing cardiac surgery. A recent meta-analysis is supporting the data published in previous years on the role of prophylactic magnesium supplementation in the prevention of postoperative atrial fibrillation (POAF) in patients undergoing cardiac surgery [33], especially when magnesium is given in the postoperative period [34]. Perioperative hypomagnesemia should have a crucial influence on cardiac adverse events after surgery [35] particularly on POAF, described as the most common cardiac complication occurred in cardiothoracic surgery [36]. The reported data on cardiac surgery patients seem to be faulty by many confounding factors. Population recruited in these search patterns are often high-risk patients or with morbid conditions that can alter magnesium homeostasis, consequently, the total circulating magnesium. In the same way, they often require additional intraoperative therapies and different surgical techniques. Furthermore, the ubiquitous role that magnesium has in biological functions makes it difficult to identify all the interconnections between magnesium homeostasis and the causes of perioperative adverse events magnesium correlated. Although further investigation is needed and the mechanisms involved are unclear, magnesium plays a role in the incidence of cardiac complications and perioperative mortality [37]. The work of Whittaker et al. [37] pointed out that perioperative changes in serum magnesium are independently predictive for 30-day mortality and cardiac morbidity on patients undergoing unplanned vascular surgery. The relevance of the date is considerable, but the authors do not address the issue of the physiopathology of surgical related hypomagnesemia, nor examine the effects of magnesium supplements on patient outcomes suggesting further high-quality research to probe the effects of the biologically active form of magnesium, its measurement and its usefulness in the perioperative setting.

The intravenous administration of magnesium has proven to be safe in any case $[33,38]$, and it has already been used as an effective therapy in the torsade de pointes [39]. The effectiveness of magnesium supplementation in the prevention of postoperative supraventricular arrhythmias seems to be the same for thoracic surgery [40]. Considering the already approved uses of magnesium, some international companies do not underestimate the results obtained until now in preventing perioperative adverse events especially in the cardiothoracic and vascular surgery and recommend the prophylactic use of intravenous magnesium for high-risk patients [41].

Less consistent results relate to the effect of additional magnesium in the perioperative non-cardiac setting. A recent meta-analysis reported magnesium prophylaxis's ineffectiveness in reducing the incidence of arrhythmias after non-cardiac surgery and no impact on mortality [42]. It is right to underline that patients included in this meta-analysis are underwent different types of non-cardiac surgery with significant heterogeneity in the trials recruited. The efficacy of additional serum magnesium to prevent intra and postoperative arrhythmic events is often compared, as in this case, with antiarrhythmics or vasoactive drugs steadily administered for the pre-existing pathology in the general population candidate for surgery. Do not measure the ionized magnesium to be replenished and, therefore, the useful proportion of serum magnesium, it may not be helpful but, on the contrary, can create confusion and lack of reliability in the date. Several drugs commonly used, including diuretics and proton-pump inhibitors, interfere with the magnesium homeostasis. There are many different factors related to the patient or pre-existing diseases that can affect the absorption, metabolism, and excretion of magnesium [43]. 
In the same way, it can be misleading to consider only the dose of magnesium administered for all patients despite the promising results, without considering the sufficient serum concentrations achieved. According to recent work, intravenous magnesium at a dose of $50 \mathrm{mg} / \mathrm{Kg}$ is associated with significantly modulated hemodynamic response during laparoscopic surgery, reducing diastolic pressure and heart rate [44]. The reason for choosing a specific intravenous magnesium dosage, as reported in the meta-analysis in question, which includes 4 RCTs, remains unknown.

Some studies evaluated the achievement of stable magnesium levels after bolus intravenous administration, how it is usually used, or followed by continuous infusion that is demonstrated the same effective levels[45]. The oral route has been suggested in prophylactic therapy to prevent postoperative arrhythmias [46], but these investigations have aimed at measurement on total magnesium and not ionized bioactive free form [47].

Although no guidelines exist for the prophylactic use of magnesium in non-cardiac surgery and the research procedures implemented to achieve such important results are still unclear, a recent survey shows that, about almost a thousand doctors interviewed, 35\% regularly use magnesium in anesthesia with a variable dosage based on personal choices [48]. Perioperative use of magnesium is also reported in the literature as an analgesic adjuvant during anesthesia or administered to reduce the demand of anesthetic drugs. Used for the pain control after laparoscopic cholecystectomy, intravenous magnesium is effective in reducing early pain within 24 hours and the requirement of anesthetic drugs [49]. This meta-analysis, with low heterogeneity and favorable outcome on pain control, have referred intravenous doses of magnesium from $7.5 \mathrm{mg} / \mathrm{Kg}$ to $50 \mathrm{mg} / \mathrm{Kg}$ or in association with continuous infusion among all trials considered. The same efficacy with various doses is reported in a systematic review investigating the effects of the supplement of magnesium in orthopedic surgery. The reported dose varied among studies considered but all within $50 \mathrm{mg} / \mathrm{kg}$ considering the maximum safe dose [50].

Other useful results on the control of postoperative pain emerge from the use of magnesium combined with local epidural anesthetic in different types of non-cardiac surgery in another series of trials evaluated [51]. Even in this case, there is a large dosage range of magnesium without serum detection. Ionized Magnesium has been forgotten once again.

Considering the rule of magnesium as a non-competitive antagonist of the $N$-methyl-d-aspartate (NMDA) receptor and its possible action on nociceptive modulation in the spinal cord [52], for several years, has been attempted to demonstrate its effectiveness on the extension of the action of intrathecal local anesthetics and opioids. Summarising the date of several RCTs, Morrison et al. suggest the advantage of adding intrathecal magnesium to lipophilic opioids with or without local anesthetics to prolong the duration of spinal anesthesia in all types of surgery except for the obstetric surgery, encouraging the implementations of other trials [53]. An interesting scientific debate has emerged about the safety and the right methods to perform a trial without neglecting the possible neurotoxicity of the intrathecal magnesium. While some authors assert safe and efficacious intrathecal magnesium dosages from 50 to $100 \mathrm{mg} / \mathrm{kg}$ without reported complications [54], others consider it possibly dangerous and thinking there is not currently licensed for intrathecal administration [55]. We add that to avoid side effects, safety levels can be established when they are accurately measured in the blood regardless of the administration route. 
The evidence that magnesium is an adjuvant in the control of postoperative pain emerges forcefully. The reduction of the opioid request and the prolonged analgesia are also obtained by the administration of the magnesium when associated with the local anesthetics in the peripheral blocks [56]. Whatever is the route of administration, intravenous, peridural, intrathecal, or peripheral, the level of adequate active magnesium is not detected, the limit for the onset of toxicity has not yet been proven and the difficulty of performing reliable trials remains.

\section{Discussion}

Magnesium represents the second cation present in the intracellular environment and the fourth most abundant in the whole organism. It plays a crucial role in the synthesis of nucleic acids and proteins, in the activity of hundreds of enzymes and is fundamental in neuromuscular contractility and the generation of the cardiac action potential [57]. Ionized serum magnesium is the only marker that specifically identifies critically ill patients with impaired magnesium status. Monitoring circulating ionized magnesium is to be preferred as a measure of bioavailability after supplementation of magnesium compared with total magnesium, and it reduces the risk of $\mathrm{Mg}$ toxicity in patients treated for $\mathrm{Mg}$ deficiency. To increase the reliability of measurement, iMg must be directly measured, and it cannot be calculated. Even though critically ill patients are one of the most exposed to a dysmagnesemia, $\mathrm{iMg}$ is infrequently measured in this clinical setting. Specialized equipment is needed for $\mathrm{iMg}$ measurement, and it requires an immediate analysis of whole blood. The examiners must consider individual-level factors, such as $\mathrm{pH}$ and serum calcium, and sample storage methods that can consistently overestimate the results. Serum detection of iMg corresponds with the biologically functional part of magnesium compared with total magnesium that instead does not differentiate between protein-bound, ligand-bound, and ionized magnesium. From our considerations, iMg is the preferable clinical biomarker of magnesium status in critically ill patients that can avoid overtreatment and

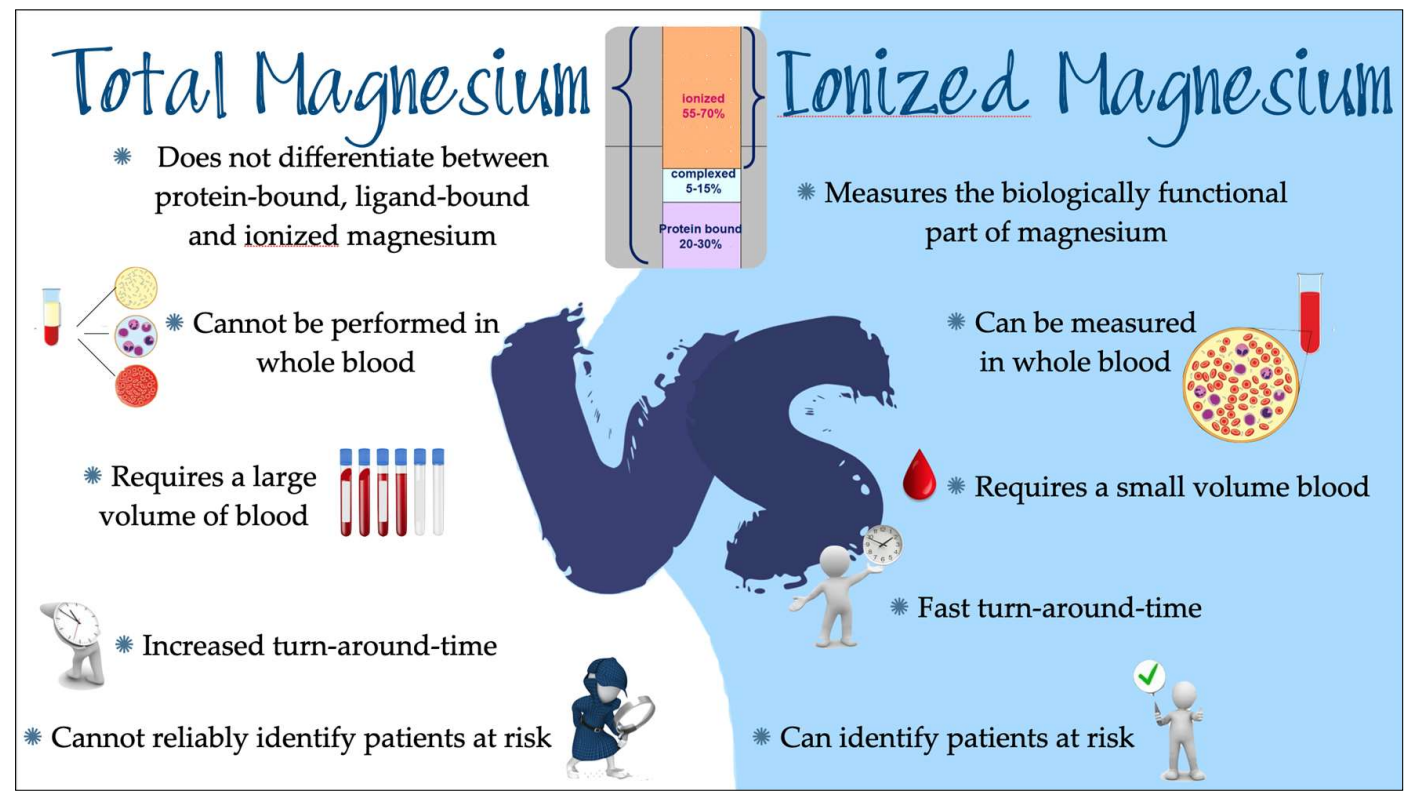

identify patients with true dysmagnesemia with a higher risk of death (Figure 1) 
Figure 1 Total Magnesium vs Ionized Magnesium. Why in our opinion we should measure ionized magnesium.

Improving the outcomes and reducing the mortality rate of surgical patients is the aim of the researches; we reported that tested add of magnesium in the perioperative period. Ionized magnesium could play an essential role in defining the optimal levels to maintain in the blood and avoid the high rate of magnesium doses using the different administrations' routes. We reported intravenous doses of magnesium sulfate range from $7.5 \mathrm{mg} / \mathrm{Kg}$ to $50 \mathrm{mg} / \mathrm{Kg}$ in different RCTs or a $50 \mathrm{mg} / \mathrm{Kg}$ to 100 $\mathrm{mg} / \mathrm{Kg}$ dose of intrathecal magnesium sulfate without a specific justification when choosing one over another. And the same goes for the epidural route with variability of doses until 500 mg of magnesium sulfate by a continuous infusion or addiction of just under half of this dose in ultrasound-guided TAP-block that has a significant effect on postoperative pain control. The role of magnesium addiction by a different administration route is clearly proven to be efficient in prolonging analgesia duration, reduces postoperative analgesic drug requirement, and influences hemodynamics responses positively during laparoscopic surgery. There are still no indications or recommendations on the use of magnesium in perioperative pain management, and the safe blood threshold has not been established. None of the reported trials have detected circulating magnesium before or after its administrations or measuring iMg despite the right results.

The date is relevant to the use of magnesium supplements in surgical patients, especially in cardiothoracic surgery, to prevent cardiac adverse events, improve outcomes, and reduce the perioperative mortality rate. The most evident results are on the prevention of supraventricular arrhythmias in high-risk patients undergoing cardiac surgery. Perioperative hypomagnesemia is described, especially in cardiac surgical patients. They are often high-risk patients with pre-existing conditions or arising in the perioperative period that can alter magnesium homeostasis. They often require additional intraoperative drugs and different surgical techniques or extracorporeal circulation that interfere with the magnesium homeostasis. While some scientific societies suggest the use of magnesium in cardiothoracic surgery, no guidelines exist for the prophylactic use of magnesium in non-cardiac surgery. The correlation between total magnesium and ionized magnesium in these clinical settings has not been tested, and more research does not use iMg measurement to correlate the safe of addiction of magnesium to the improvement of outcomes. Despite the real benefit of magnesium addiction in reduced mortality and the proven underdiagnosed hypomagnesemia in critically ill patients and the perioperative setting, too few researches are investing in the trials that well-characterized the optimal concentrations of iMg concerning prevent health outcomes and avoid toxicity effects.

The adult clinical ranges reported of total serum magnesium are from 0,7 to $1 \mathrm{mmol} / \mathrm{L}$, with slight variations proposed in the literature often narrow range from 0,75 to 0,95 [58]. It is already clear that the reference ranges presented and currently in use for total magnesium derived from many studies of magnesium detection in healthy groups with public health consensus. Many authors still consider total serum $\mathrm{Mg}$ as a useful biomarker of the state of magnesium but only because it is the only test currently used in clinical practice at a reasonable cost and with standardized methodologies. Reference values validating reflect the possibility to measure total serum magnesium, urinary magnesium, and fractional excretion with procedures extensively utilized in clinical laboratories and, therefore, readily available for clinical research. Changes in the total serum magnesium concentration, easily 
detectable, was correlated with the extracellular fraction of magnesium considered in balance with the various compartments involved in magnesium homeostasis. But total magnesium measurement in serum does not reflect intracellular concentrations or the free portion available; it also doesn't consider the percentage bounds to proteins, other water molecules, and the dynamic flow across compartments to maintain homeostasis. The results obtained on the correlation between magnesium status and pathologies, organ failures, and clinical outcomes of ill subjects are still unclear with different analytical methods used for detections [59]. In practice, changes in total magnesium from the validated ranges may not correspond to magnesium's active form's bioavailability. Even an amount within the field may falsely indicate a bioavailability of active form that does not exist. With values above the proposed range, there is a real possibility that the individual is already impaired in biologically active magnesium with a latent deficiency [58].

Why not measure the active form directly then? Reference ranges have been proposed for ionized $\mathrm{Mg}$ from 0.54 to $0.67 \mathrm{mM} / \mathrm{L}$ with an average interval of just 130 micromolar with a portion of ionized $\mathrm{Mg}$ that exceeds even $70 \%$ of the total circulating magnesium. However, the evidenced-based data are still deficient in obtaining unanimous consent [60]. Ionized magnesium refers to free magnesium ions, and their detection requires laboratory techniques other than those for the total $\mathrm{Mg}$. The availability of magnesium ion-selective electrode and specific sensor technology linked with commercial blood analyzers makes possible more reliable and widespread ionized magnesium measurements in a clinical setting [61]. Allowing data collection in clinical practice directly on patients considering all those conditions that can alter magnesium levels as independent factors, interfering with intake, absorption, losses, and excretion can create sufficient data to assume reference levels based on the evidence. iMg can be detected in whole blood, minimizing the execution times, and waiting for results compared to the serum samples for $\mathrm{tMg}$ detection. The measurement can be influenced by other ions, especially calcium, by the temperature, $\mathrm{pH}$, or dilution of the sample [62], corrected by the operators' calibration and correct sampling. The absence of detection of $\mathrm{iMg}$ in clinical practice seems to derive from a lack of standardization of the procedure rather than by cost but, above all, the absence of correlation between the values of ionized magnesium measured and the quantity to be replenished to optimize magnesium levels it does not create requirements for researchers for getting over the impasse.

\section{Conclusions}

Hypomagnesemia (and Hyper-) are present in several clinical manifestations, including cardiovascular indication and sepsis. Accurate and routinely measuring ionized magnesium in critically ill patients is necessary to replace the active physiological part. Measure and monitor the biologically active form of magnesium and establish an adequate circulating threshold is still a current challenge. The specialized equipment required to measure the biologically active way instead of circulating total magnesium does the research demanding work. Most studies do not consider serum magnesium measurement at all but only after supplementation in the surgical patients. The inclusion of iMg in the critical care profile enables rapid assessment of the physiologically active and clinically relevant magnesium fraction. Dosage, administration route, and optimal timing in the perioperative setting 
should be clarified concerning the measured levels accurately when it is reasonable to give magnesium supplements to achieved clinical benefits. Future studies are needed to stabilize the normal range of ionized magnesium.

Conflicts of Interest: The authors declare no conflict of interest.

\section{References}

1. Hardwick, L.L.; Jones, M.R.; Buddington, R.K. et al. Comparison of calcium and magnesium absorption: in vivo and in vitro studies. The American journal of physiology 1990 Nov;259(5 Pt 1):G720-6

2. Koch, S.M.; Warters, R.D.; Mehlhorn U. The simultaneous measurement of ionized and total calcium and ionized and total magnesium in intensive care unit patients. Journal of Critical Care, 2002, vol 17, No 3, 203205

3. Barbagallo, M.; Dominguez, L.J.; Galioto, A.; Pineo, A.; Belvedere, M. Oral magnesium supplementation improves vascular function in elderly diabetic patients. Magnes. Res. 2010, 23, 131-137

4. Kazaks, A.G.; Uriu-Adams, J.Y.; Albertson, T.E.; Shenoy, S.F.; Stern, J.S. Effect of oral magnesium supplementation on measures of airway resistance and subjective assessment of asthma control and quality of life in men and women with mild to moderate asthma: A randomized placebo-controlled trial. J. Asthma 2010, 47, 83-92.

5. Zavaczki, Z.; Szollosi, J.; Kiss, S.A.; Koloszar, S.; Fejes, I.; Kovacs, L.; Pal, A. Magnesium-orotate supplementation for idiopathic infertile male patients: A randomized, placebo-controlled clinical pilot study. Magnes. Res. 2003, 16, 131-136

6. Costello, R.B.; Nielsen, F. Interpreting magnesium status to enhance clinical care: Key indicators. Curr. Opin. Clin. Nutr. Metab. Care 2017, 20, 504-511; Glasdam, S.M.; Glasdam, S.; Peters, G.H. The Importance of Magnesium in the Human Body: A Systematic Literature Review. Adv. Clin. Chem. 2016, 73, 169-193.

7. Markendeyulu, D.; Benhar, N.V.A. Study of prevalence of hypomagnesemia and clinical outcome in critically ill patients in tertiary care center in Andhra Pradesh. J. Evid. Based Med. Healthc. 2019; 6(7), 464-467.

8. Bharath, M.S.; Udayashankar, R.; Hiregoudar. On Admission Hypomagnesemia and its Adverse Effects in Critically Ill Patients Admitted to ICU in Tertiary Care Centre. International Journal of Contemporary Medical Research 2016, Volume 3, Issue 9, ICV: 50.43

9. Jiang, P.; Lv, Q.; Lai, T.; Xu, F. Does Hypomagnesemia Impact on the Outcome of Patients Admitted to the Intensive Care Unit? A Systematic Review and Meta-Analysis. Shock. 2017;47(3):288-295.

10. Limaye, C.S.; Londhey, V.A.; Nadkar, M.Y.; et al. Hypomagnesemia in critically ill medical patients. J Assoc Physicians India. 2011;59:19-22.

11. Velissaris, D.; Karamouzos, V.; Pierrakos, C.; Aretha, D.; Karanikolas, M. Hypomagnesemia in Critically Ill Sepsis Patients. Journal of clinical medicine research, (2015), 7(12), 911-918. https://doi.org/10.14740/jocmr2351w

12. Beheshti, S.; Tabatabaei, S.; Jahangirifard, A.; Safarzaei, M.; Golestani Eraghi, M.; Mahjoubifard, M. Effects of magnesium on clinical outcome of critical care traumatic patients. Journal of Cellular \& Molecular Anesthesia (JCMA), 2017 2. doi:10.22037/jcma.v2i1.13579

13. Upala, S.; Jaruvongvanich, V.; Wijarnpreecha, K.; Sanguankeo, A. Hypomagnesemia and mortality in patients admitted to intensive care unit: a systematic review and meta-analysis. QJM : monthly journal of the Association of Physicians, 2016 109(7), 453-459. https://doi.org/10.1093/qjmed/hcw048

14. Cheungpasitporn, W.; Thongprayoon, C.; Qian, Q. Dysmagnesemia in Hospitalized Patients: Prevalence and Prognostic Importance. Mayo Clinic proceedings, 2015, 90(8), 1001-1010. https://doi.org/10.1016/j.mayocp.2015.04.023

15. Hansen, B. A.; Bruserud, Ø. Hypomagnesemia in critically ill patients. Journal of intensive care, 2018, 6, 21. https://doi.org/10.1186/s40560-018-0291-y

16. Del Gobbo, L. C.; Imamura, F.; Wu, J. H.; de Oliveira Otto, M. C.; Chiuve, S. E.; Mozaffarian, D. Circulating and dietary magnesium and risk of cardiovascular disease: a systematic review and meta-analysis of prospective studies. The American journal of clinical nutrition, 2013, 98(1), 160-173. https://doi.org/10.3945/ajcn.112.053132 
17. Chen, M.; Sun, R.; Hu, B. Zhonghua wei zhong bing ji jiu yi xue, 2015 , 27(3), $213-217$. https://doi.org/10.3760/cma.j.issn.2095-4352.2015.03.011

18. Chernow, B.; Bamberger, S.; Stoiko, M.; Vadnais, M.; Mills, S.; Hoellerich, V. Hypomagnesemia in patients in postoperative intensive care. Chest 1989;95:391-7.

19. Rubeiz, G.J.; Thill-Baharozian, M.; Hardie, D.; Carlson, R.W. Association of hypomagnesemia and mortality in acutely ill medical patients. Crit Care Med 1993;21:203-9.

20. Safavi, M.; Honarmand, A. Admission hypomagnesemia - Impact on mortality or morbidity in critically ill patients. Middle East J Anesthesiol 2007;19:645-60.

21. Guérin, C.; Cousin, C.; Mignot, F.; Manchon, M.; Fournier, G. Serum and erythrocyte magnesium in critically ill patients. Intensive Care Med 1996;22:724-7.

22. Soliman, H.M.; Mercan, D.; Lobo, S.S.; Mélot, C.; Vincent, J.L. Development of ionized hypomagnesemia is associated with higher mortality rates. Crit Care Med 2003;31:1082-7.

23. Dabbagh, O.C.; Aldawood, A.S.; Arabi, Y.M.; Lone, N.A.; Brits, R.; Pillay, M. Magnesium supplementation and the potential association with mortality rates among critically ill non-cardiac patients. Saudi Med J 2006;27:821-5.

24. Celi, L. A.; Scott, D. J.; Lee, J.; Nelson, R.; Alper, S. L.; Mukamal, K. J.; Mark, R. G.; Danziger, J. Association of hypermagnesemia and blood pressure in the critically ill. Journal of hypertension, $2013,31(11), 2136-$ 2141. https://doi.org/10.1097/HJH.0b013e3283642f18

25. Escuela, M.P.; Guerra, M.; Añón, J.M. Total and ionized serum magnesium in critically ill patients. Intensive Care Med. 2005;31(1):151-156.)

26. Kyriacou, L. Monitoring of serum total and ionised magnesium in patients with acute myocardial infarction. Australian Journal of Medical Science, 2008, 29, 84-90.

27. Yeh, D.D.; Chokengarmwong, N.; Chang, Y. Total and ionized magnesium testing in the surgical intensive care unit - Opportunities for improved laboratory and pharmacy utilization. J Crit Care. 2017;42:147-151.

28. Huijgen, H.; Soesan, M.; Sanders, R.; Mairuhu, W.; Kesecioglu, J.; Sanders, G. Magnesium Levels in Critically Ill Patients What Should We Measure? American journal of clinical pathology, 2000 114, 688-695.

29. Lorentz, M.; Vianna, B. Disritmias cardíacas e anestesia. Revista Brasileira de Anestesiologia, 2011 61, 805813. doi:10.1590/S0034-70942011000600013

30. Zafar, M.S.; Wani, J.I.; Karim, R.; Mir, M.M.; Koul, P.A. Significance of serum magnesium levels in critically ill patients. Int J Appl Basic Med Res. 2014, 4(1):34-37

31. Panahi, Y.; Mojtahedzadeh, M.; Najafi, A. The role of magnesium sulfate in the intensive care unit. EXCLI J. 2017;16:464-482. Published 2017 Apr 5.

32. Jolly, K.; Faulconer, R.; McEwan, R. The incidence of hypomagnesemia following abdominal aortic aneurysm surgery. Ann R Coll Surg Engl 2015;97:379-81.

33. Fairley, J.L.; Zhang, L.; Glassford, N.J. Magnesium status and magnesium therapy in cardiac surgery: A systematic review and meta-analysis focusing on arrhythmia prevention. Crit Care. 2017; 42: 69-77.

34. Chaudhary, R.; Garg, J.; Turagam, M.; Chaudhary, R.; Gupta, R.; Nazir, T.; Bozorgnia, B.; Albert, C.; Lakkireddy, D. Role of Prophylactic Magnesium Supplementation in Prevention of Postoperative Atrial Fibrillation in Patients Undergoing Coronary Artery Bypass Grafting: a Systematic Review and Meta-Analysis of 20 Randomized Controlled Trials. Journal of atrial fibrillation, 2019, 12(1), 2154. https://doi.org/10.4022/jafib.2154

35. Jolly, K.; Faulconer, R.; McEwan, R.; Becker, H.; Garnham, A. The incidence of hypomagnesaemia following abdominal aortic aneurysm surgery. Annals of the Royal College of Surgeons of England, 2015, 97(5), 379381. https://doi.org/10.1308/003588415X14181254790004

36. D'Agostino, R.S.; Jacobs, J.P.; Badhwar, V.; Fernandez, F.G.; Paone, G.; Wormuth, D.W. The Society of Thoracic Surgeons adult cardiac surgery database: 2018 update on outcomes and quality. Ann Thorac Surg. 2018;105(1):15-23. doi: 10.1016/j.athoracsur.2017.10.035

37. Whittaker, J. D.; Downes, F.; Becker, H.; Garnham, A.; Wall, M. Influence of Perioperative Serum Magnesium for Cardiac and Noncardiac Morbidity and Mortality Following Emergency Peripheral Vascular Surgery. Journal of cardiothoracic and vascular anesthesia, 2019, 33(2), 474-479. https://doi.org/10.1053/j.jvca.2018.05.042

38. Salaminia, S.; Sayehmiri, F.; Angha, P.; Sayehmiri, K.; Motedayen, M. Evaluating the effect of magnesium supplementation and cardiac arrhythmias after acute coronary syndrome: A systematic review and metaanalysis. BMC Cardiovascular Disorders, 2018, 18. doi:10.1186/s12872-018-0857-6 
39. Duff, J. P.; Topjian, A.; Berg, M. D.; Chan, M.; Haskell, S. E.; Joyner, B. L.; Jr, Lasa, J. J.; Ley, S. J.; Raymond, T. T.; Sutton, R. M.; Hazinski, M. F.; Atkins, D. L. 2018 American Heart Association Focused Update on Pediatric Advanced Life Support: An Update to the American Heart Association Guidelines for Cardiopulmonary Resuscitation and Emergency Cardiovascular Care. Circulation, 2018, 138(23), e731-e739. https://doi.org/10.1161/CIR.0000000000000612

40. Panchal, A. R.; Berg, K. M.; Hirsch, K. G.; Kudenchuk, P. J.; Del Rios, M.; Cabañas, J. G.; Link, M. S.; Kurz, M. C.; Chan, P. S.; Morley, P. T.; Hazinski, M. F.; Donnino, M. W. 2019 American Heart Association Focused Update on Advanced Cardiovascular Life Support: Use of Advanced Airways, Vasopressors, and Extracorporeal Cardiopulmonary Resuscitation During Cardiac Arrest: An Update to the American Heart Association Guidelines for Cardiopulmonary Resuscitation and Emergency Cardiovascular Care. Circulation, 2019, 140(24), e881-e894. https://doi.org/10.1161/CIR.0000000000000732

41. Macle, L.; Cairns, J.; Leblanc, K.; Tsang, T.; Skanes, A.; Cox, J. L.; Healey, J. S.; Bell, A.; Pilote, L.; Andrade, J. G.; Mitchell, L. B.; Atzema, C.; Gladstone, D.; Sharma, M.; Verma, S.; Connolly, S.; Dorian, P.; Parkash, R.;Talajic, M.; Nattel, S.; CCS Atrial Fibrillation Guidelines Committee. 2016 Focused Update of the Canadian Cardiovascular Society Guidelines for the Management of Atrial Fibrillation. The Canadian journal of cardiology, 2016, 32(10), 1170-1185. https://doi.org/10.1016/j.cjca.2016.07.591

42. Oesterle, A.; Weber, B.; Tung, R.; Choudhry, N. K.; Singh, J. P.; Upadhyay, G. A. Preventing Postoperative Atrial Fibrillation After Noncardiac Surgery: A Meta-analysis. The American journal of medicine, 2018, 131(7), 795-804.e5. https://doi.org/10.1016/j.amjmed.2018.01.032

43. Gröber U. Magnesium and Drugs. International journal of molecular sciences, 2019, 20(9), 2094. https://doi.org/10.3390/ijms20092094

44. Zhang, J.; Wang, Y.; Xu, H.; Yang, J. Influence of magnesium sulfate on hemodynamic responses during laparoscopic cholecystectomy: A meta-analysis of randomized controlled studies. Medicine, 2018, 97(45), e12747. https://doi.org/10.1097/MD.0000000000012747

45. Biesenbach, P.; Mårtensson, J.; Osawa, E.; Eastwood, G.; Cutuli, S.; Fairley, J.; Matalanis, G.; Bellomo, R. Magnesium supplementation: Pharmacokinetics in cardiac surgery patients with normal renal function. Journal of critical care, 2018, 44, 419-423. https://doi.org/10.1016/j.jcrc.2018.01.011

46. Jannati, M.; Shahbazi, S.; Eshaghi, L. Comparison of the Efficacy of Oral versus Intravascular Magnesium in the Prevention of Hypomagnesemia and Arrhythmia after CABG. Brazilian journal of cardiovascular surgery, 2018, 33(5), 448-453. https://doi.org/10.21470/1678-9741-2018-0070

47. Glasdam, S. M.; Glasdam, S.; Peters, G. H. The Importance of Magnesium in the Human Body: A Systematic Literature Review. Advances in clinical chemistry, 2016, 73, 169-193. https://doi.org/10.1016/bs.acc.2015.10.002

48. Cavalcanti, I. L.; de Lima, F.; da Silva, M.; da Cruz Filho, R. A.; Braga, E.; Verçosa, N. Use Profile of Magnesium Sulfate in Anesthesia in Brazil. Frontiers in pharmacology, 2019, 10, 429. https://doi.org/10.3389/fphar.2019.00429

49. Chen, C.; Tao, R. The Impact of Magnesium Sulfate on Pain Control After Laparoscopic Cholecystectomy: A Meta-Analysis of Randomized Controlled Studies. Surgical laparoscopy, endoscopy \& percutaneous techniques, 2018, 28(6), 349-353. https://doi.org/10.1097/SLE.0000000000000571

50. Peng, Y. N.; Sung, F. C.; Huang, M. L.; Lin, C. L.; Kao, C. H. The use of intravenous magnesium sulfate on postoperative analgesia in orthopedic surgery: A systematic review of randomized controlled trials. Medicine, 2018, 97(50), e13583. https://doi.org/10.1097/MD.0000000000013583

51. Li, L.-Q.; Fang, M.-D.; Wang, C.; Lu, H.-L.; Wang, L.-X.; Xu, H.-Y.; Zhang, H.-Z. Comparative evaluation of epidural bupivacaine alone and bupivacaine combined with magnesium sulfate in providing postoperative analgesia: a meta-analysis of randomized controlled trials. BMC anesthesiology, 2020, 20, 39. doi:10.1186/s12871-020-0947-8

52. Yaksh, T.; Fisher, C.; Hockman, T.; Wiese, A. Current and Future Issues in the Development of Spinal Agents for the Management of Pain. Current Neuropharmacology, 2016, 14. doi:10.2174/1570159X14666160307145542

53. Morrison, A.; Hunter, J.; Halpern, S.; Banerjee, A. Effect of intrathecal magnesium in the presence or absence of local anaesthetic with and without lipophilic opioids: A systematic review and meta-analysis. British journal of anaesthesia, 2013, 110. doi:10.1093/bja/aet064

54. Pascual-Ramírez, J.; Gil-Trujillo, S.; Alcantarilla, C. Intrathecal magnesium as analgesic adjuvant for spinal anesthesia: a meta-analysis of randomized trials. Minerva anestesiologica, 2013, 79(6), 667-678. 
55. Albrecht, E.; Kern, C.; Kirkham, K. R. The safety profile of neuraxial magnesium has not been properly addressed. British journal of anaesthesia, 2014, 112(1), 173-174. https://doi.org/10.1093/bja/aet450

56. Abd-Elsalam, K. A.; Fares, K. M.; Mohamed, M. A.; Mohamed, M. F.; El-Rahman, A.; Tohamy, M. M. Efficacy of Magnesium Sulfate Added to Local Anesthetic in a Transversus Abdominis Plane Block for Analgesia Following Total Abdominal Hysterectomy: A Randomized Trial. Pain physician, 2017, 20(7), 641-647.

57. Baker W. L. Treating arrhythmias with adjunctive magnesium: identifying future research directions. European heart journal. Cardiovascular pharmacotherapy, 2017, 3(2), 108-117. https://doi.org/10.1093/ehjcvp/pvw028.

58. Costello, R. B.; Elin, R. J.; Rosanoff, A.; Wallace, T. C.; Guerrero-Romero, F.; Hruby, A.; Lutsey, P. L.; Nielsen, F. H.; Rodriguez-Moran, M.; Song, Y.; \& Van Horn, L. V. Perspective: The Case for an Evidence-Based Reference Interval for Serum Magnesium: The Time Has Come. Advances in nutrition (Bethesda, Md.), 2016 , 7(6), 977-993. https://doi.org/10.3945/an.116.012765

59. Glasdam, S. M. The Importance of Magnesium in the Human Body: A Systematic Literature Review. Advances in Clinical Chemistry 2016 Elsevier Inc. ISSN 0065-2423. doi.org/10.1016/bs.acc.2015.10.002

60. Altura, B. T. Importance of Ionized Magnesium Measurements in Physiology and Medicine and the Need for Ion-selective Electrodes. Journal of Clinical Case Studies ( ISSN 2471-4925 ), 2016 1. doi:10.16966/24714925.111

61. Zhang, W. Point Of Care Testing of Ionized Magnesium in Blood with Potentiometric Sensors - Opportunities and Challenges. American Journal of Biomedical Sciences, 2011, 3, 301-312. doi:10.5099/aj110400301

62. Ben Rayana, M.; Burnett, R.; Covington, A.; D'Orazio, P.; Fogh-Andersen, N.; Jacobs, E.; John, A. Guidelines for sampling, measuring and reporting ionized magnesium in undiluted serum, plasma or blood: International Federation of Clinical Chemistry and Laboratory Medicine (IFCC): IFCC Scientific Division, Committee on Point of Care Testing. Clinical chemistry and laboratory medicine : CCLM / FESCC, 2005, 43, 564-569. doi:10.1515/CCLM.2005.098 